\title{
Anatomy and radiology of the variations of aortic arch branches in 1,266 patients
}

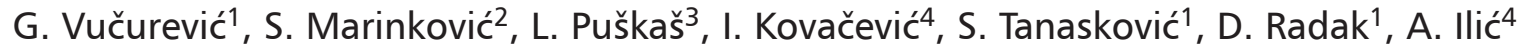 \\ ${ }^{1}$ Vascular Surgery Clinic, Dedinje Cardiovascular Centre, Faculty of Dentistry, Pancevo, Serbia \\ 2Institute of Anatomy, Faculty of Medicine, University of Belgrade, Belgrade, Serbia \\ ${ }^{3}$ Neurosurgical Clinic, Faculty of Medicine, University of Belgrade, Belgrade, Serbia \\ ${ }^{4}$ Clinic of Ophthalmology, Faculty of Medicine, University of Belgrade, Belgrade, Serbia
}

[Received 5 December 2012; Accepted 18 January 2013]

Background: The most reliable data about arterial variations, which are very important in surgery and radiology, can be obtained from a large series of patients. Materials and methods: We examined angiographic and multislice computerised tomography (MSCT) images in a group of 1,265 patients and in 1 dissected specimen. Results: While in 946 (74.72\%) of the patients a normal vascular pattern (type I) was noticed, in the remaining 320 (25.28\%) patients variations of the branches of the aortic arch were found, which were classified into types II through VIII and a few subtypes. Type /I (2.84\%) comprised a common origin of the left common carotid and subclavian arteries. Type III (15.56\%) was related to an origin of the left subclavian artery from the brachiocephalic trunk. Type IV (0.55\%) included the aortic origin of both common carotid and subclavian arteries, with the right subclavian artery having a retroesophageal course. Type $V(0.24 \%)$ included the same 4 supra-aortic branches, which, however, arose from a double or a right-sided aortic arch. Type VI (3.63\%) comprised the aortic origin of the left vertebral artery, type VII (0.24\%) the same origin of the right vertebral artery, and type VIII $(2.22 \%)$ the aortic origin of the thyroidea ima artery. A corresponding embryological background and clinical implications of the described aberrant vessels were presented.

Conclusions: In more than one quarter of the cases, the branching pattern of the examined arteries did not follow the classical pattern. Detailed knowledge of aortic branch variations is of great significance in anatomy, embryology, and clinical medicine, especially in radiology and thoracic surgery. (Folia Morphol 2013; 72, 2:113-122)

Key words: anatomical variations, aortic arch, neck arteries, radiology, thoracic arteries, thoracic surgery

\section{INTRODUCTION}

Variations of the thoracic arteries, including branches of the aortic arch, are interesting from an anatomical and embryological aspect $[4,8,27,32]$. They are, however, more important for thoracic operations, especially in vascular, cardiac, oesophageal, and mediastinal surgery $[1,9,12,14,34]$. Modern radiological techniques, especially computerised tomographic angiography (CTA), magnetic resonance angiography (MRA), multislice CT (MSCT), and MSCT angiography, have enabled an elegant insight into the thoracic blood vessels $[21,27]$. Obviously, the mentio- 
ned variations are also very important in radiological diagnostics and interventional radiology.

The aim of this study was, firstly, to present the anatomical variations of the aortic arch and the supra-aortic arteries in a very large group of patients following transfemoral angiography, MSCT imaging, or MSCT angiographic examination. Secondly, to present the embryological background of those variations. And thirdly, to stress the radiological and surgical significance of the variations based on the obtained anatomical and radiological data.

\section{MATERIALS AND METHODS}

A group of 1,265 patients were examined following approval by the Ethics Committee of the University Clinical Centre. The group consisted of 751 males and 514 females, with mean ages of 68 and 65 years, respectively. The angiography was performed by a transfemoral approach after Seldinger in all individuals in order to identify the arterial variations and their possible vascular diseases, as well as for certain measurements of the relevant vessels, especially their diameter. Selected patients underwent CT, MSCT, or MSCT angiography. One case of aberrant origin of the vertebral artery, obtained during a routine dissection for students' practice, was included into the patient group. Thus, the total number of patients was 1,266.

In addition to angiography and other radiological procedures, all patients underwent the relevant biochemical analysis, cardiovascular and neurologic examination. Statistical methods comprised counting of the percentages related to the whole series of patients $(1,266)$, as well as to a group with the arterial variations (320).

\section{RESULTS}

The normal (left) arch of the aorta (Fig. 1) typically gives rise to 3 large vessels: the brachiocephalic trunk (BCT), or the innominate artery, which divides into the right common carotid (RCCA) and subclavian arteries (RSCA), as well as the left common carotid (LCCA) and subclavian arteries (LSCA). The common carotid arteries divide into the right internal (RICA) and external carotid arteries (RECA), and into the same vessels on the opposite side (the LICA and LECA). Direct branches of the aortic arch are also known as the primary or the supra-aortic arteries. We examined both the primary and secondary arteries, as well as the variations of the aortic arch itself.

Our study was carried out in 1,266 patients. Among them, 946 (74.72\%) showed a normal vascular pattern,

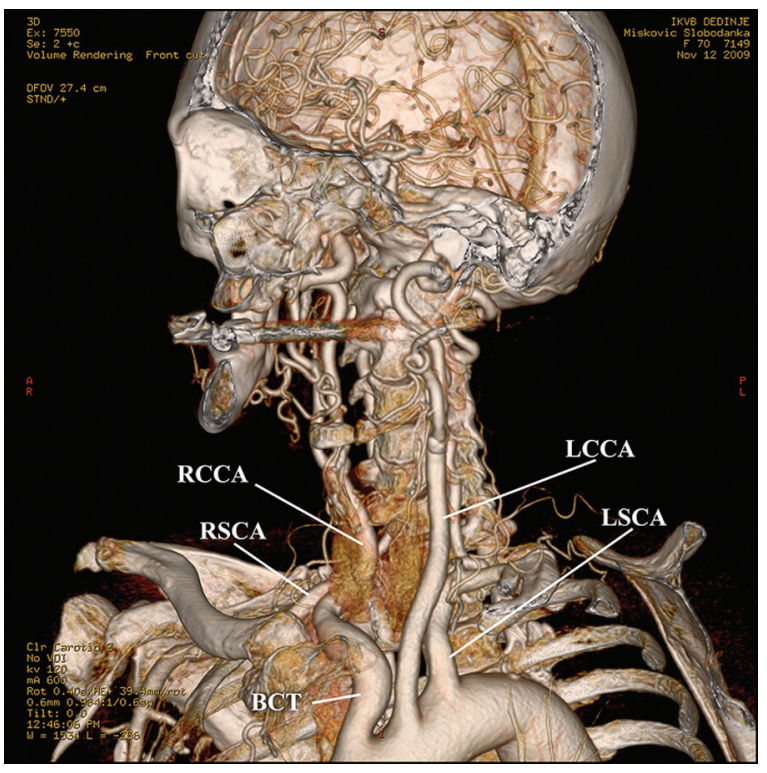

Figure 1. A multislice computed tomographic image to show a normal branching pattern (type I) of the arch of the aorta. BCT brachiocephalic trunk; RCCA and LCCA — right and left common carotid arteries; RSCA and LSCA — right and left subclavian arteries.

whereas 320 (25.28\%) presented with variations of the supra-aortic vessels and the aortic arch.

\section{Aortic arch variations}

Examination of the aortic arch itself revealed a double arch and several right-sided arches. A double aortic arch, which was found in a male patient $(0.08 \%)$, encircled and compressed the trachea and oesophagus, and showed a separate origin of the main arteries: the RCCA and RSCA, and the LCCA and LSCA, respectively.

A right-sided aortic arch was found in 4 male patients $(0.32 \%)$. In 2 of them it compressed the trachea or oesophagus. The descending aorta in 3 patients continued along the right side, but in the remaining person it was found to return to the left side of the mediastinum. Branches of the right-sided arch were arranged in a 4-vessel pattern. Namely, the BCT was absent, so that the RCCA and RSCA arose directly from the aortic arch, in addition to a normal aortic origin of the LSCA and LCCA (see later).

\section{Patterns of the aortic arch branches}

The branches of the aortic arch were classified into 8 types (Table 1 ). In some of these groups we made a combination of the variations of both the aortic arch and its branches. In addition to the classical vascular pattern (type I), the remaining 7 types showed 
Table 1. Normal origin and variations of the aortic arch branches

\begin{tabular}{lcccc}
\hline $\begin{array}{l}\text { Types of } \\
\text { variants }\end{array}$ & $\begin{array}{c}\text { No. of } \\
\text { patients }\end{array}$ & $\begin{array}{c}\text { Frequency (\%): } \\
\text { all patients }\end{array}$ & $\begin{array}{c}\text { No. of } \\
\text { patients }\end{array}$ & $\begin{array}{c}\text { Frequency (\%): } \\
\text { patients with variants }\end{array}$ \\
\hline $\mathrm{I}^{*}$ & $946^{*}$ & $74.72^{*}$ & 0 & 0.00 \\
II & 36 & 2.84 & 36 & 11.25 \\
IIla & 86 & 6.79 & 86 & 26.88 \\
IIIb & 84 & 6.64 & 84 & 26.25 \\
IIIc & 27 & 2.13 & 27 & 8.44 \\
IV** & 7 & 0.55 & 7 & 2.19 \\
Va*** & 1 & 0.08 & 1 & 0.31 \\
Vb** & 1 & 0.08 & 1 & 0.31 \\
Vc** & 1 & 0.08 & 37 & 0.31 \\
Vla & 37 & 2.92 & 8 & 11.56 \\
Vlb & 8 & 0.63 & 1 & 2.50 \\
Vlc & 1 & 0.08 & 3 & 0.31 \\
VII & 3 & 0.24 & 28 & 0.93 \\
VIII & 28 & 2.22 & 320 & 8.76 \\
Total & 1,266 & 100.00 & 100.00 \\
\hline
\end{tabular}

${ }^{*}$ patients lacking arterial variations; ${ }^{* *}$ some variations associated with a right-sided aortic arch; ***a variation associated with a double aortic arch

a specific origin, arrangement, and relationship of the mentioned branches.

Type I. This type, which was found in 946 patients (74.72\%), refers to the usual branching pattern: the BCT, LCCA, and LSCA (Fig. 1). These vessels arose from a normal (left) aortic arch in all the patients, except in the 5 individuals mentioned with a double or a right-sided aortic arch. We measured diameters of the supra-aortic branches and their divisions, i.e. the RICA and RECA, and LICA and LECA (Table 2).

The BCT, which averaged $0.6 \mathrm{~cm}$ in diameter (Table 2) and between 2.2 and $7.1 \mathrm{~cm}$ (mean, $4.6 \mathrm{~cm}$ ) in length, divided into the RCCA and RSCA above $(94.71 \%)$, below $(1.97 \%)$, or at the level (3.32\%) of the sternoclavicular joint. The RCCA gave rise to the superior thyroid artery in 11 patients $(0.87 \%)$ before dividing into the RICA and RECA, most often at the level of the $4^{\text {th }} 5^{\text {th }}$ cervical vertebrae. The RCCA was usually smaller than the RSCA (Table 2).

The LCCA was similar to the RCCA, except for its longer course (Fig. 1). It gave off the superior thyroid artery in 143 patients (11.29\%). Its terminal branches, i.e. the LICA and LECA, also arose most frequently at the mentioned level. The former artery was seen to give rise to the ascending pharyngeal artery in 32 patients (2.53\%). The LECA was similar to the RECA. It gave off a linguofacial trunk in
Table 2. Measurements of the relevant arteries in type I (946 patients)

\begin{tabular}{llc}
\hline $\begin{array}{l}\text { Artery: } \\
\text { type (side) }\end{array}$ & & $\begin{array}{c}\text { Diameter: } \\
\text { mean }[\mathrm{cm}]\end{array}$ \\
\hline Brachiocephalic trunk & (BCT right) & 2.1 \\
Common carotid artery & (RCCA) & 0.7 \\
& (LCCA) & 0.6 \\
Internal carotid artery & (RICA) & 0.3 \\
& (LICA) & 0.4 \\
External carotid artery & (RECA) & 0.4 \\
& (LECA) & 0.4 \\
Subclavian artery & (RSCA) & 0.8 \\
& (LSCA) & 0.7 \\
Vertebral artery & (RVA) & 0.2 \\
& (LVA) & 0.3 \\
\hline
\end{tabular}

BCT — brachiocephalic trunk; RCCA and LCCA — right and left common carotid arteries; RECA and LECA — right and left external carotid arteries; RSCA and LSCA — right and left subclavian arteries; RVA and LVA — right and left vertebral artery

$0.39 \%$ of the patients. The LCCA also was smaller than the LSCA.

Types II through VIII of the supra-aortic branches were distinguished within a group of 320 patients (25.28\%). Each type is presented separately (Table 1).

Type II. Type II contained, in fact, only 2 branches of the aortic arch, i.e. the $\mathrm{BCT}$, as well as the same origin 


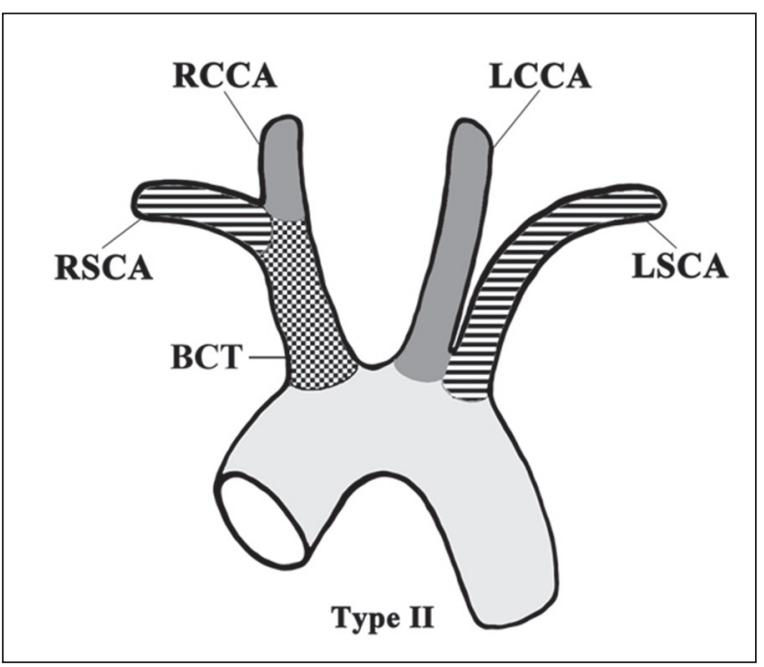

Figure 2. A drawing presenting a typical brachiocephalic trunk (BCT) and a common origin of the left carotid (LCCA) and subclavian artery (LSCA); RCCA — right common carotid artery; RSCA — right subclavian artery.

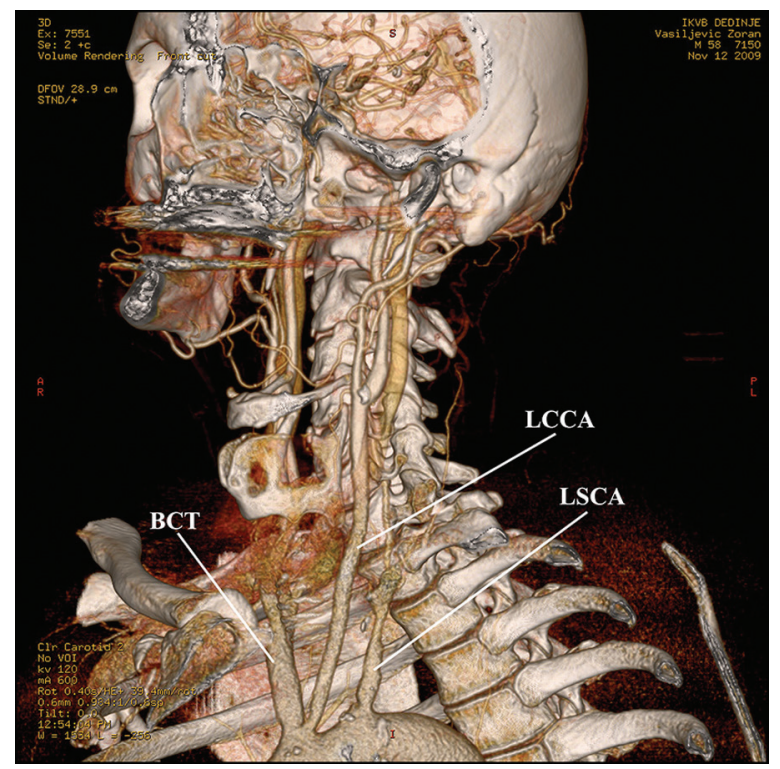

Figure 3. A multislice computed tomographic image of type Illa. The left common carotid artery (LCCA) shares a origin site with the brachiocephalic trunk (BCT). Note the left subclavian artery (LSCA).

site of the LCCA and LSCA (Fig. 2). This double BCT pattern was present in 36 patients (2.84\%) (Table 1).

Type III. This type, which was observed in $15.56 \%$ of the patients, was presented with 3 variants (Table 1). In the first variant, or subtype IIIa, the LCCA and BCT shared the same site of origin in 86 patients (Fig. 3). In the IIIb subtype, observed in 84 patients, the LCCA originated from a typical BCT (Figs. 4A, B). In the Illc variant, the LCCA arose from a shorter BCT in
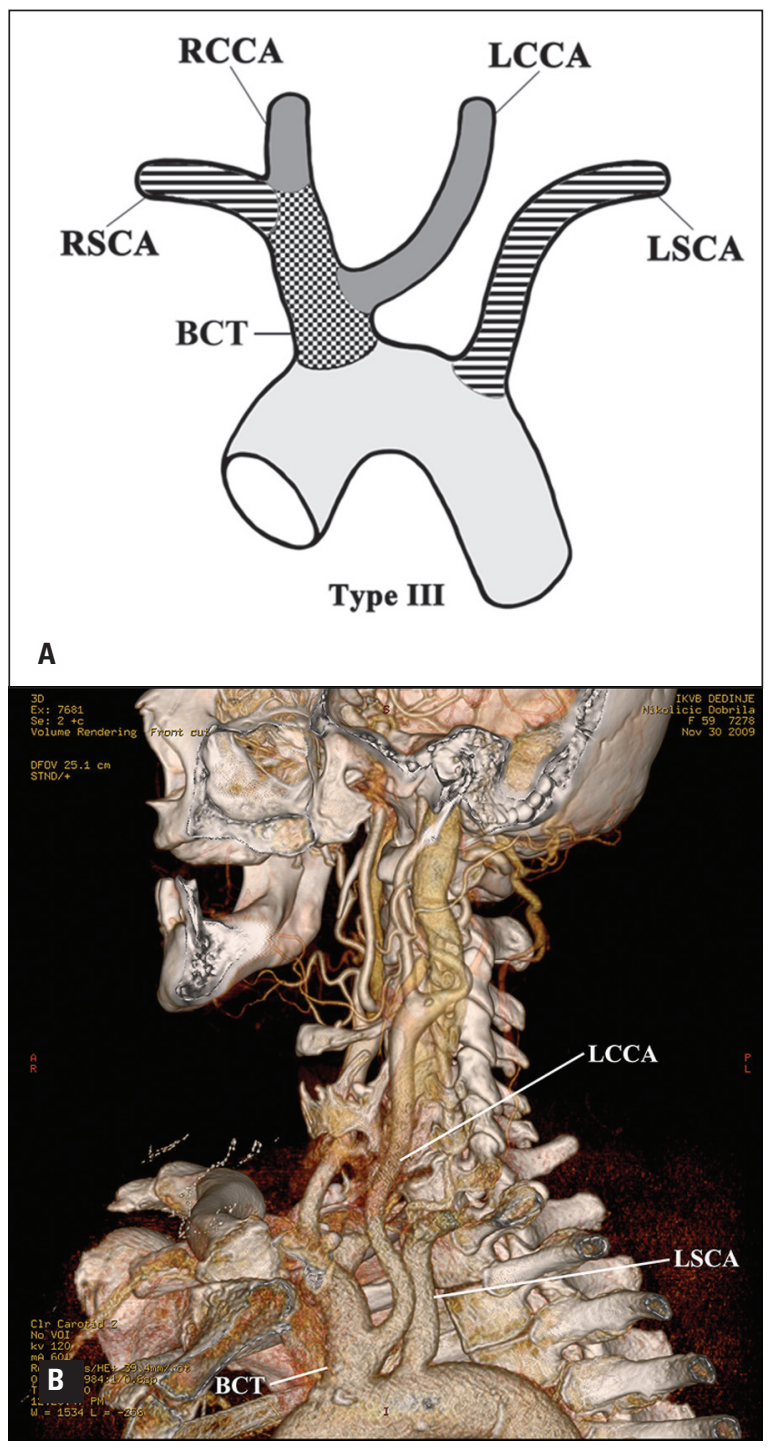

Figure 4. A drawing (A) and a multislice computed tomographic image (B) of type Illb. The left common carotid artery (LCCA) originates from the brachiocephalic trunk (BCT), close to the left subclavian artery (LSCA); RCCA — right common carotid artery; RSCA — right subclavian artery.

27 patients. The LSCA in all the subgroups originated from the aortic arch as the most distal branch.

Type IV. Type IV comprised the aortic origin of both carotid arteries (the RCCA and LCCA) and the subclavian arteries (RSCA and LSCA). This 4-vessel pattern was present in 7 patients, i.e. in 5 with a left aortic arch (Fig. 5A) and in the remaining 2 with a right-sided arch (Fig. 5B). All of them had the RSCA as a distal aortic branch. This so-called arteria lusoria extended posterior to the esophagus. The initial segment of the arteria lusoria was enlarged, i.e. the LSCA originated from a Kommerell diverticulum. 


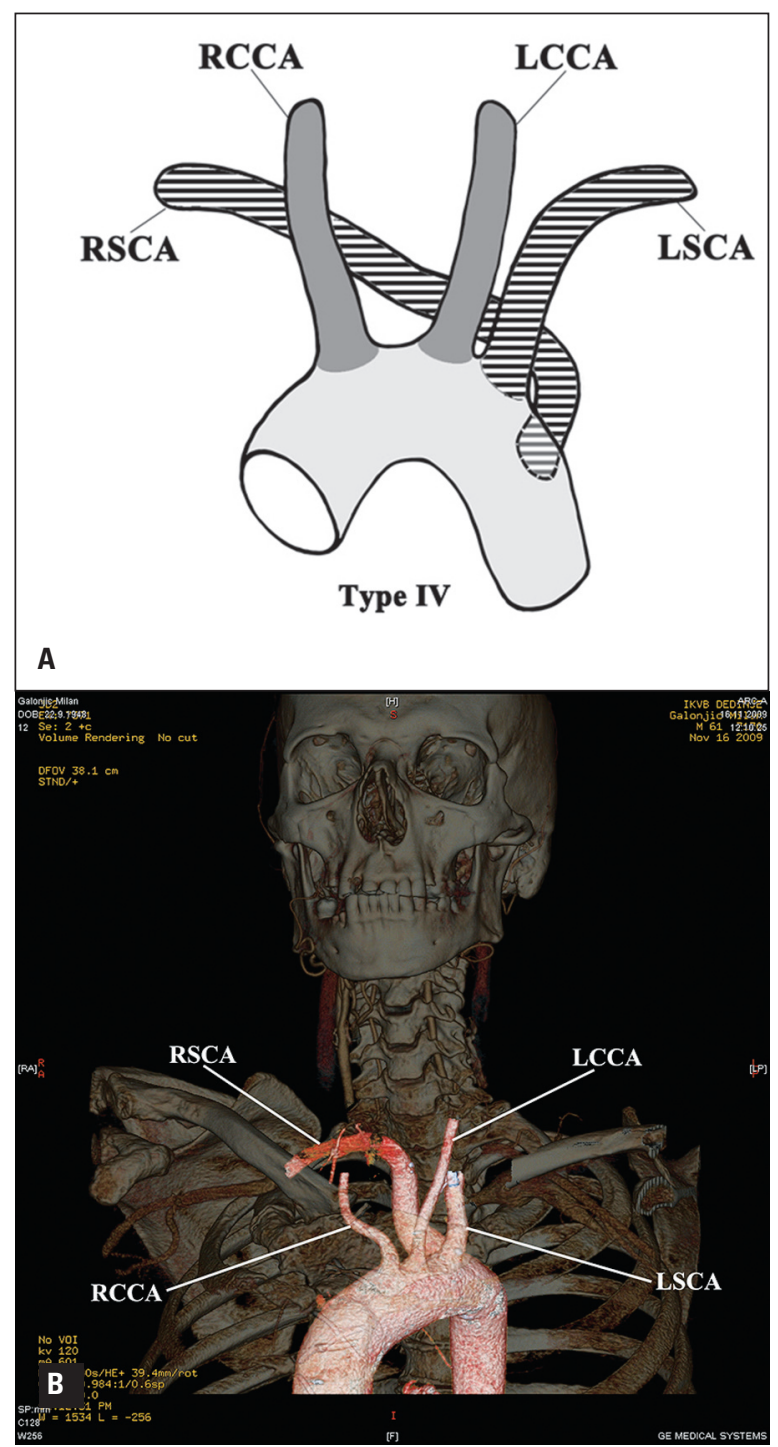

Figure 5. A drawing (A) and a multislice computed tomographic image (B) of type IV with the right subclavian artery (RSCA) arising as an arteria lusoria in patients with a left-sided $(\mathbf{A})$ and a right-sided arch of the aorta (B); LSCA — left subclavian artery; LCCA and RCCA — left and right common carotid arteries.

Type V. This was also a 4-vessel pattern (the RCCA, LCCA, RSCA and LSCA), which, however, comprised 3 subtypes in 3 patients (Table 1). In 1 with a double aortic arch (type Va), the RCCA and RSCA arose on the right side, and the LCCA and LSCA on the left side. Another patient, with a right-sided arch, showed the LSCA and RSCA as the most distal aortic branches (type Vb) (Fig. 6A), while in the remaining patient with a right arch the LSCA and RSCA were the most proximal branches (type Vc) (Fig. 6B). All the 4 vessels in the latter case had longer initial parts than usual.

Type VI. Type VI is characterised by an aberrant origin of the left vertebral artery (LVA) in 3.63\% of the
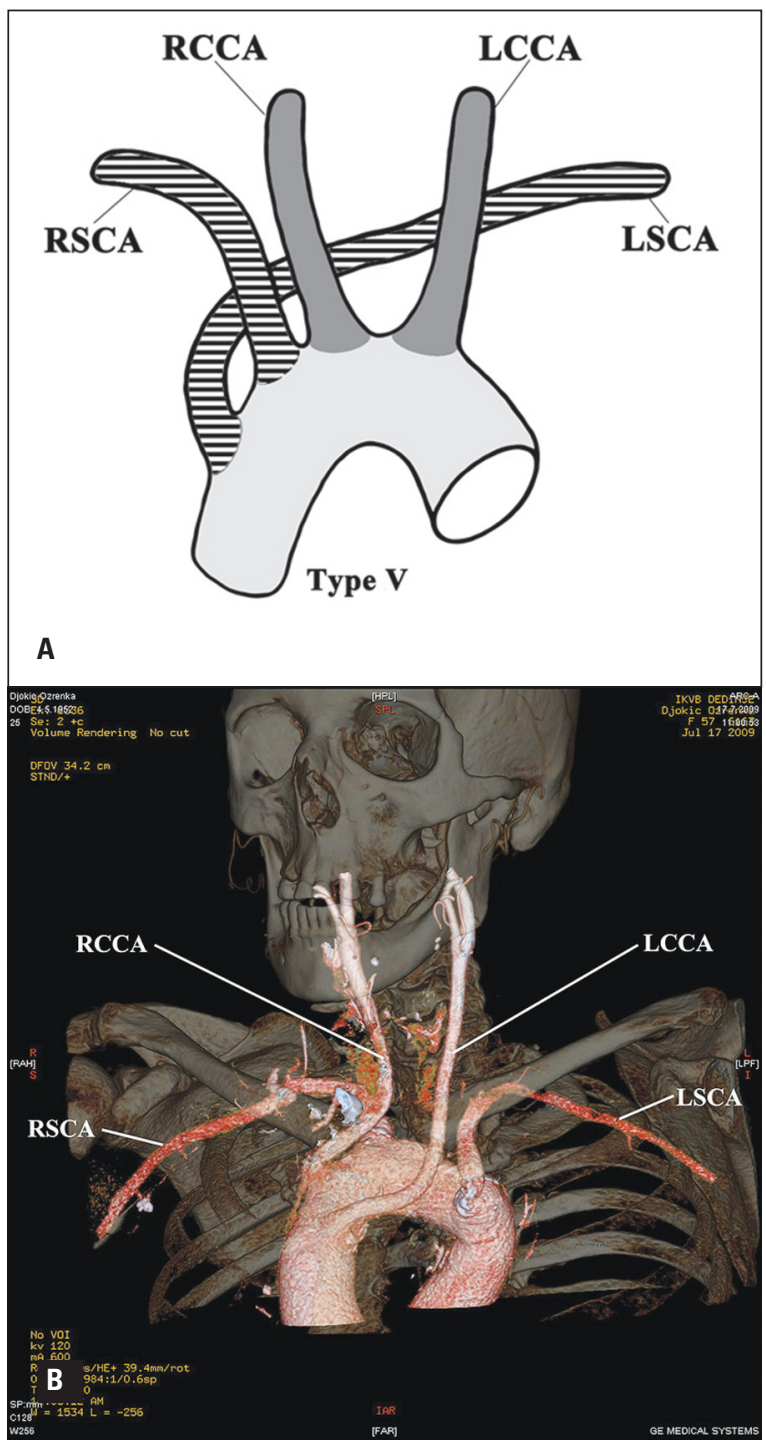

Figure 6. A drawing (A) and a multislice computed tomographic image (B) of type $\mathrm{Vb}$. A separate origin of the right and left subclavian arteries (RSCA and LSCA), and the right and left common carotid arteries (RCCA and LCCA) in patients with a right-sided aortic arch.

patients. It also presented with 3 subtypes (Table 1). The most frequent 1 was the Vla subtype, which showed a typical vessel arrangement (the BCT, LCCA, LSCA) with the LVA origin between the LCCA and LSCA (Figs. 7A, B). The LVA in the Vlb subtype was a component of a 5-vessel pattern: the RSCA, RCCA, LCCA, LVA, and LSCA. The VIc subtype is referred to the LVA as the most distal branch, as was seen in our anatomical specimen (Fig. 8), which was included into the patient group.

Type VII. Type VII showed the aortic origin of the right vertebral artery (RVA) in 3 patients (Table 1). In all the subjects the RVA was the most distal branch (Fig. 9). The RVA continued to the right side by coursing be- 


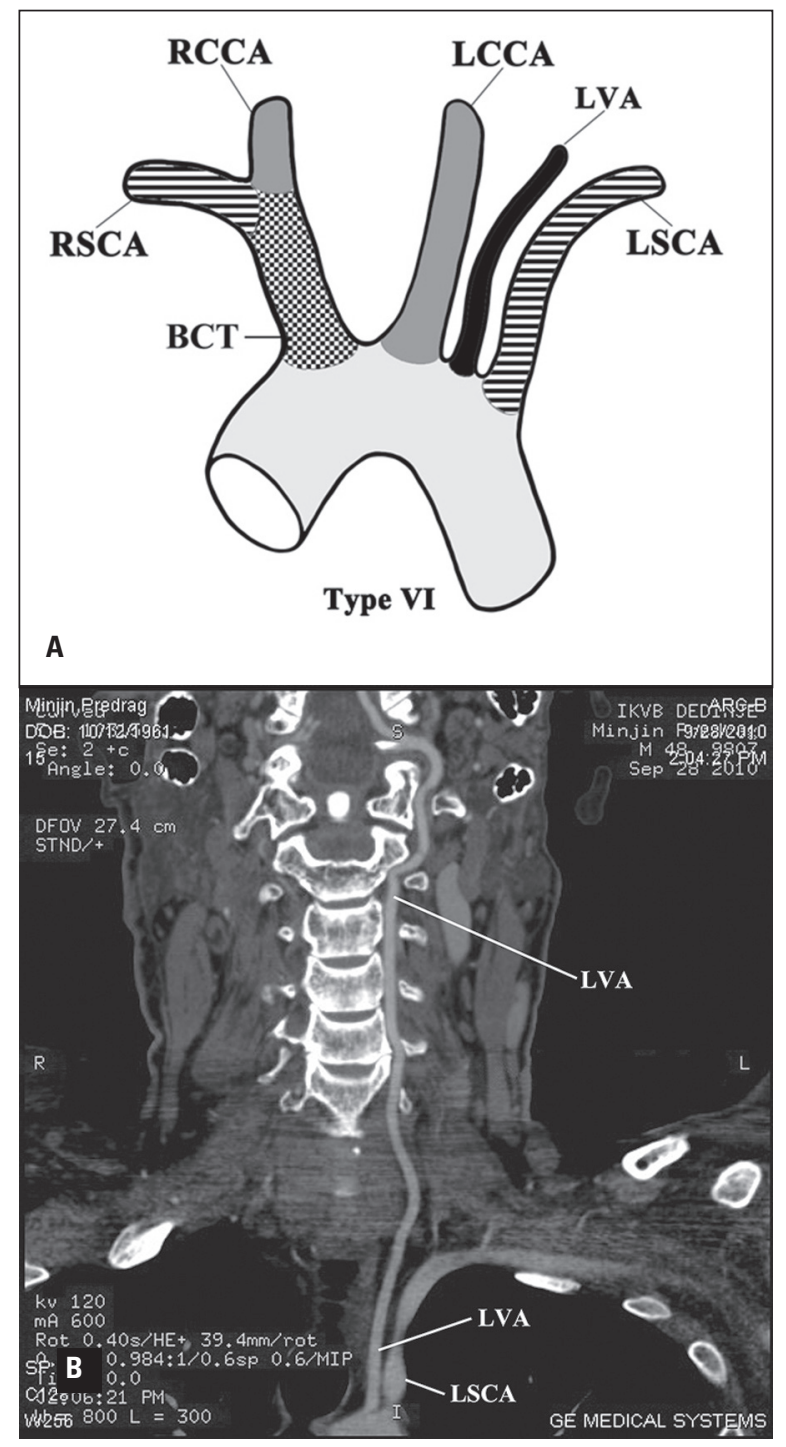

Figure 7. A drawing (A) and an anterior multislice computed tomographic angiogram (B) presenting type Vla, i.e. the left vertebral artery (LVA) arising from the aortic arch just proximal to the left subclavian artery (LSCA); rest abbreviations as in Table 2.

tween the trachea and oesophagus. This type in our study belongs to the 4-vessel pattern.

Type VIII. Type VIII comprised a typical vascular pattern (BCT, LCCA, LSCA) (Fig. 10), but also the aortic origin of the thyroidea ima artery (ThIA). The latter vessel arose between the BCT and LCCA in $2.22 \%$ of our patients (Table 1).

Some subjects from both groups, i.e. from 320 patients with variations and from 946 of the remaining individuals, presented with certain clinical signs due to a corresponding vascular pathology of the supra-aortic arteries or their branches: compression to the adjacent organs, arterial dissection, stenosis, occlusion, aneurysm, elongation, kinking, or coiling.

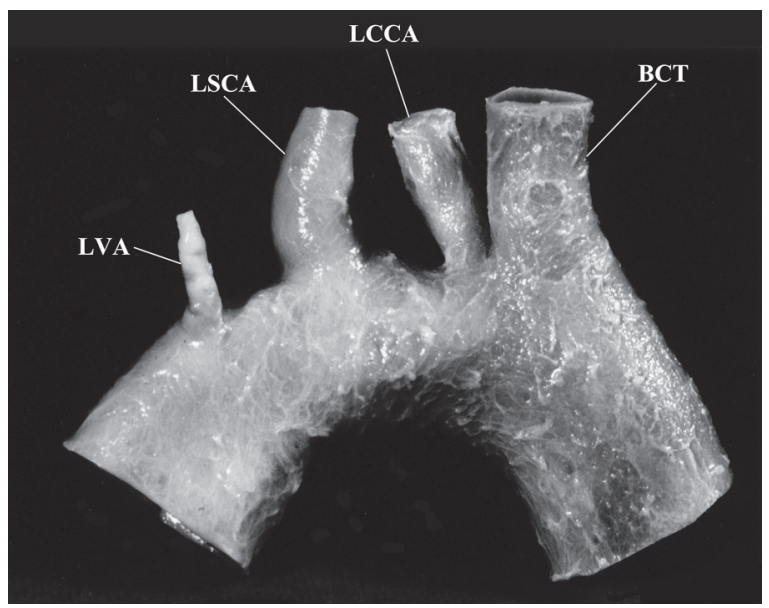

Figure 8. An anatomical specimen in a posterior view showing type Vlb, i.e. the aortic origin of the left vertebral artery (LVA) distal to the left subclavian artery (LSCA), the left common carotid artery (LCCA), and the brachiocephalic trunk (BCT).

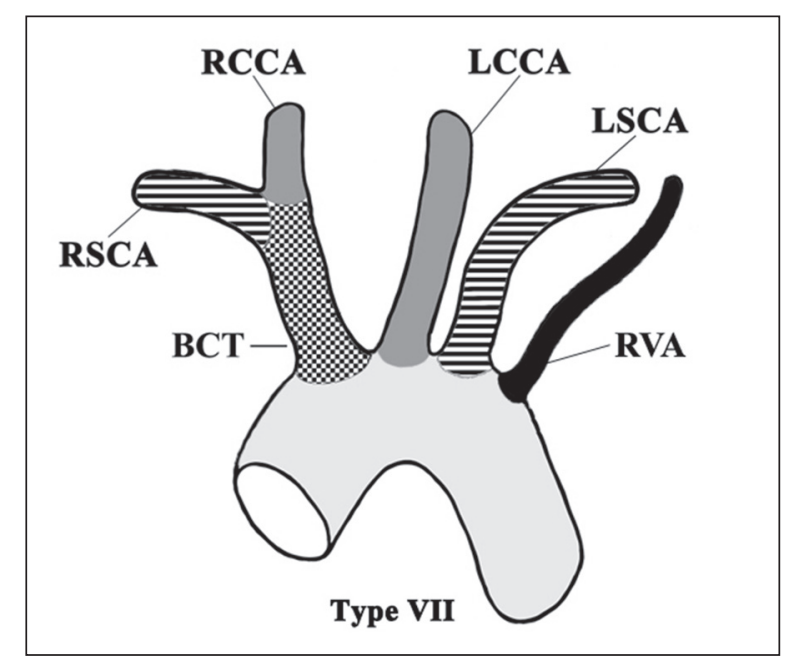

Figure 9. A drawing presenting the aortic origin of the right vertebral artery (RVA); rest abbreviations as in Table 2.

\section{DISCUSSION}

Among the 1,265 patients examined, plus one anatomical specimen, the variations of the branches of the aortic arch were revealed in 320 individuals (25.28\%) These variations have been presented by some other authors but in much smaller groups of patients or anatomical specimens $[4,27,28]$. The anomalies of the aortic arch and its branches arise as a result of altered development of primitive aortic arches (Figs. 11A, B), or may occur as a consequence of irregular and imperfect development of the aorticopulmonary septum of the truncus arteriosus $[4,23,32]$. 


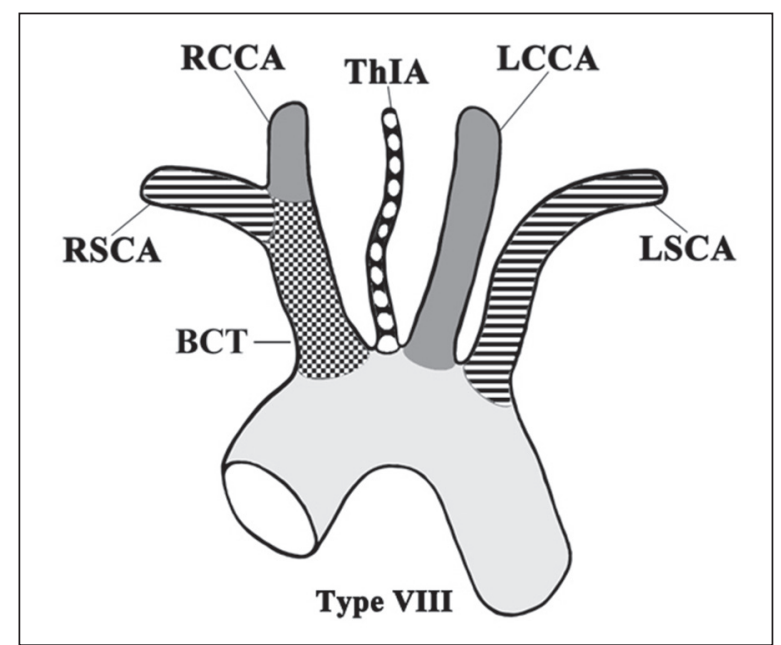

Figure 10. A drawing showing the aortic origin of the thyroidea ima artery (ThIA); rest abbreviations as in Table 2.

\section{Variation types}

The variations from our study were classified into 7 types, taking into account both the branches and the aortic arch itself.

Type I. The common vascular pattern was present in $74.72 \%$ of our patients, while it was observed in a range between $64.9 \%$ and $94.3 \%$ in other series [27, 28]. This 3-vessel pattern of the aortic arch branches understands the presence of the BCT, LCCA, and LSCA. The $B C T$ is localised on the right, and extremely rarely on the left side. In the latter case, the BCT normally divides into the RCCA and RSCA, which soon return to the right side of the neck [31]. Nevertheless, type I develops embryologically in a typical manner (Fig. 11B).

The mentioned aortic arch vessels usually do not give off the side branches in the mediastinum, but occasionally the BCT may give rise to the internal thoracic, inferior thyroid, or right vertebral artery [31]. On the other hand, the LCCA may give off the superior thyroid artery (in $11.29 \%$ of our patients), and very rarely the inferior thyroid, thyroidea ima, laryngeal, or left vertebral arteries [27, 32].

Type II. This pattern comprises a left BCA, in addition to the present right $B C A[26,28]$. It is a result of an involution of part of the left $4^{\text {th }}$ embryonic arch (Fig. 11B) [9]. Consequently, the LCCA arises in common with the LSCA. Since such a double BCT pattern is seen in birds, it is also known as the avian type [32]

Type III. This type, consisting of the LCCA arising from the $B C T$, occurs when the left horn of the aortic sack fails to develop (Fig. 11B) [28, 32]. Being similar to the vascular pattern of the cattle, this type is known as the bovine aortic arch variant $[13,27]$. In these cases, the RCCA, as one of the two BCT divisions, may give rise to the right vertebral artery $[6,8,12$, $21]$. Type III in our study was observed in $15.56 \%$, as compared to $11-27 \%$ found by other authors $[27,29]$.

Type IV. It comprises the aortic origin of all the main branches, among which the right subclavian artery is the most distal vessel (the RCCA, LCCA, LSCA, and RSCA). The latter vessel is known as the arteria lusoria $[12,25,27,28]$. It courses posterior to the trachea and oesophagus, and hence its name: the retroesophageal RSCA. However, it may also extend between the trachea and oesophagus, or in front of the trachea $[10,11,27]$. We found it in $0.55 \%$ of the cases, which is within the reported range of $0.13 \%$ through $25 \%[6,11]$.

This anomalous vessel is caused by an obliteration of the right $4^{\text {th }}$ aortic arch and a portion of the dorsal aorta proximal to the right $7^{\text {th }}$ intersegmental artery, along with persistence of the $Z$ segment of the right dorsal aorta [30, 32]. In this way, the right $7^{\text {th }}$ intersegmental artery and the $Z$ segment continues as the RSCA to the left dorsal aorta $[28,32]$. The RSCA is obviously the most distal branch. In addition, the RVA in these instances may arise from the RCCA [30].

Type $\mathbf{V}$. This type, which is also a 4-vessel pattern, is sometimes a mirror image of the previous type. The RSCA and LSCA arise either as the most distal branches (Figs. 5A, 6A) or the most proximal vessels (Fig. 6B) of a right-sided aortic arch [3, 9, 27, 28]. The aberrant LSCA in the former cases is the result of involution of the left dorsal aorta between the left subclavian and common carotid arteries, in association with persistence of the right dorsal aorta and the left dorsal aorta between the $7^{\text {th }}$ intersegmental artery and the junction with part Z (Fig. 11B). Due to that, the first aortic branch is the LCCA, followed by the RCCA, RSCA, and LSCA.

Type VI. This 4-vessel pattern (BCT, LCCA, LVA, LSCA) comprises the LVA, which has such a position in the majority of patients $[4,19]$. Type $\mathrm{VI}$ may also be a 5-vessel pattern (RSCA, RCCA, LCCA, LSCA, LVA). Nevertheless, the LVA is very rarely localised distal to the LSCA [8], as was the case in our anatomical specimen. Type $\mathrm{VI}$ was found in $3.63 \%$ of subjects in the present study, which is in accordance with other reports $(2.5-8.0 \%)[4,25,27]$.

The aberrant origin of the LVA is due to persistence of the $6^{\text {th }}$ or $8^{\text {th }}$ left intersegmental artery, respectively, 


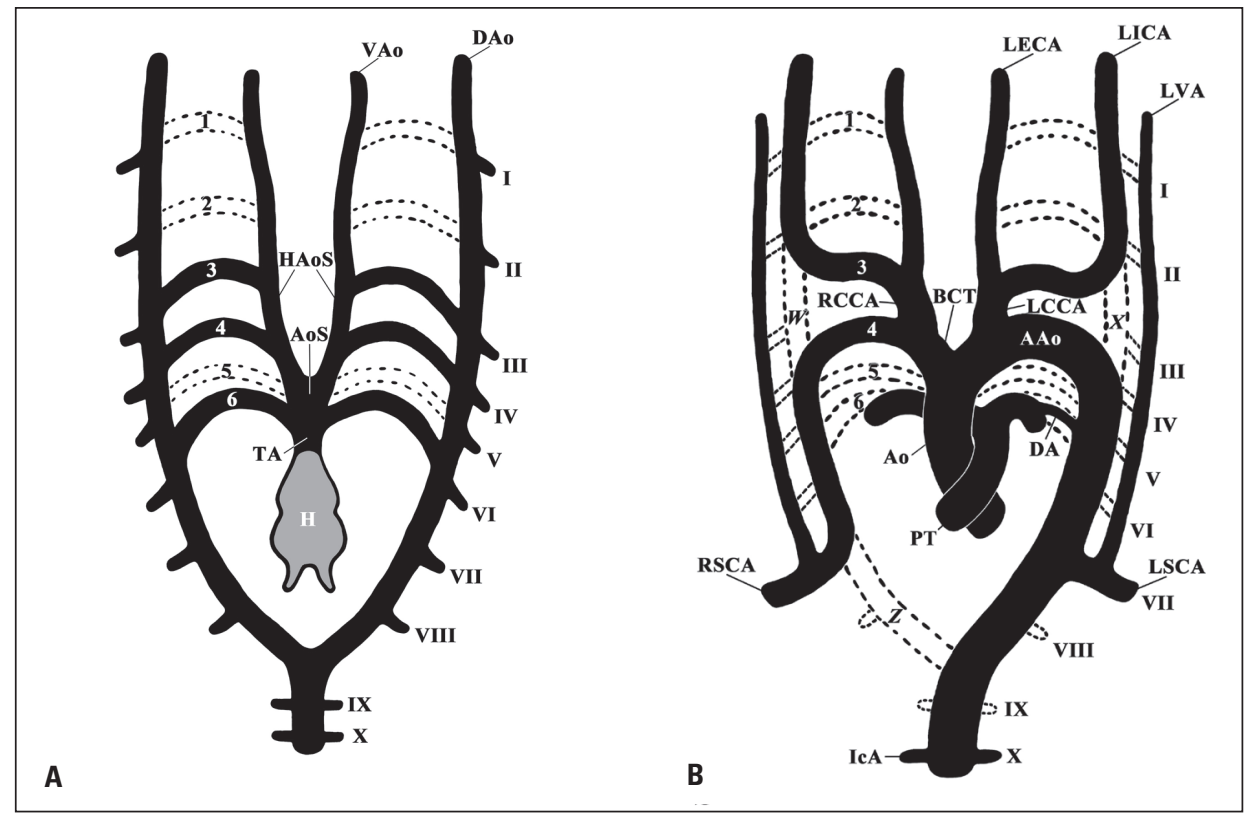

Figure 11. Embryonic development of the arterial system. A. Truncus arteriosus (TA), which leaves the heart (H), is continuous with the aortic sack (AoS) whose horns (HAoS) form the right and left ventral aortae (VAo). The aortic arches (1 through 6) interconnect the ventral and dorsal aortae (DAo). Note the cranial and thoracic intersegmental arteries (I through X); B. Development of the main arteries, i.e. the pulmonary trunk (PT) and aorta $(\mathrm{Ao})$ with its arch (AAo), interconnected by the ductus arteriosus (DA), as well as the brachiocephalic trunk (BCT), the right and left common carotid arteries (RCCA and LCCA), the right and left external carotid (LECA), and internal carotid arteries (LICA), as well as the right and left subclavian arteries (RSCA and LSCA). The left vertebral artery (LVA), like its counterpart on the right, interconnects the cranial intersegmental arteries (I through VI). Some of the remaining intersegmental vessels become the intercostal arteries (IcA). The aortic arches are labelled by numerals 1 through 6 . Note the regressed segments $(\mathrm{X}, \mathrm{W}, \mathrm{Z})$ of the dorsal aortae.

one of which becomes the initial part of the LVA [16, 28]. If the LVA develops from the $6^{\text {th }}$ artery, it will be located between the LCCA and LSCA in adults. If, however, the LVA originates from the $8^{\text {th }}$ artery, it will be situated distal to the LSCA (Fig. 11B). Finally, there is a common stem of the LSCA and LVA in some people [5, 15, 29].

Type VII. This type showed the aortic origin of the RVA, which was found in $0.24 \%$ of our patients. In general, the RVA may arise between the RSCA and RCCA, the LCCA and LSCA, or distal to the LSCA [16, 22]. Such types of origin could be due to a separate development of the RVA from the right $6^{\text {th }}$ or $8^{\text {th }}$ embryonic intersegmental artery [16].

The RVA in our patients was the most distal aortic branch. It arose from the right $8^{\text {th }}$ intersegmental artery, i.e. distal to the RSCA, after losing a connection with the latter vessel. The RVA was continuous with the persistent $Z$ part of the right dorsal aorta [17]. They both became continuous with the distal part of the left dorsal aorta (Fig. 11B). Due to that, the RVA originated distal to the LSCA in those patients.
Finally, an origin of both the LVA and RVA from the aortic arch is an extremely rare event [16, 22, 33]. One or both of the aberrant vertebral arteries may arise distal to or among the other branches of the aortic arch. The RVA in some patients originates directly from the aortic arch, whereas the LVA forms a common stem with the LSCA [15].

Type VIII. This type is characterised by the aortic origin of the ThIA, which was located between the BCT and the LCCA. This variant was present in $2.22 \%$ of our patients, as compared to the range $0.16-10.0 \%$ in other reports. The ThIA, which ascends in front of the trachea and the thyroid gland, may also arise from the BCT, RCCA, or RICA or, very rarely, from the vertebral artery $[22,27,30]$.

\section{GENERAL DISCUSSION}

Several kinds of vessel variations were noticed by the present authors and the mentioned investigators, i.e. the alterations in their size or number, the absence of some vessels, their aberrant origin or position, or both. There are often various combinations of 2 or more aberrant arteries in the same patient 
$[3,6,10,11]$. Similarly, a combination of aberrations of both branches and the aortic arch variations can be observed, especially in patients with a double or a right-sided aortic arch [1, 9, 18, 35].

By comparing the frequencies in our study, it is clear that type III, i.e. the origin of the LCCA from the $\mathrm{BCT}$, is the most frequent variation, which occurred in $15.56 \%$ of patients (Table 1). On the other hand, types IV, V, and VII are the rarest ones, with a frequency less than $1 \%$.

In general, we noticed the majority of the supra-aortic variations described thus far. We did not find, however, a common stem of the RCCA and LCCA or of the RSCA and LSCA, some other origin types of the RVA, LVA, or both, nor the aortic origin of some other aberrant vessels, e.g. the internal thoracic, thymic, or left coronary arteries $[6,27]$. On the other hand, we revealed some rare patterns, e.g. those presented in Figures $6 \mathrm{~B}$ and 8 . The mentioned differences can be explained, among others, by some national and racial features or environmental circumstances $[4,27]$.

The observed variations are of great clinical significance. Thus, a double or a right-sided aortic arch, then a common stem of the RCCA and LCCA, a left BCT, a double BCT, or a retroesophageal RSCA, may cause a compression of the trachea and the oesophagus and hence dyspnoea, cough, recurrent respiratory infections, and dysphagia $[1,3,9,11,27,34]$. In the latter case, an arterioesophageal fistula may develop, which is usually a fatal event [11].

An anomalous origin of a common carotid artery and, especially, a common trunk of the RCCA and LCCA, distal origin of the latter, or an aberrant vertebral artery, seem to be associated with a higher incidence of cerebrovascular disorders [16, 20, 28, 29]. Thrombosis of a retroesophageal subclavian artery may cause acute ischaemia of the upper limb [11]. The aortic origin of some aberrant vessels, such as the internal thoracic, thyroid, or coronary arteries, may also have certain clinical implications.

Similarly, a left BCT or a longer BCT that also inclines to the left, as well as the RCCA or the LCCA, thyroidea ima artery, and similar aberrant vessels, may extend in front of the trachea above the sternum, which can cause their injury during tracheostomy, thyroid resection, laryngeal transplantation, mediastinoscopy, etc. [28, 31]. The RVA arising from the common carotid artery can be lacerated during thyroid or anterior cervical spine surgery $[28,30]$. The arteria lusoria is important in oesophageal surgery.
The RCCA or LCCA may bifurcate into the internal and external carotid arteries very low, i.e. in the thorax, or very high - at the level of the hyoid bone or even the styloid process, which is important in thoracic, neck, and head surgery $[1,34]$. Non-recognition of certain aberrant aortic arch branches at surgery may have fatal consequences [27].

As regards vascular surgery in symptomatic patients, various techniques can be used: division of a vascular aortic ring, transposition and reimplantation of certain arteries, angioplasty, endovascular grafts, stent placement, bypass grafting, etc. [1, 2, $7,9,10,14,34]$. Radiologists usually apply various types of angiography, MSCT, catheterisation, and trans-catheter coil occlusion, either for examination and identification of the aberrant arteries or for the treatment of their pathology $[24,27]$.

\section{CONCLUSIONS}

Three kinds of the aortic arches and 8 main types of their branching patterns were revealed in this study. Left aortic arch and I and III branching patterns were the most frequent. The common and the rare variations can be accompanied by certain clinical symptoms, especially when associated with a corresponding vascular pathology. Patients with these variants are at risk of iatrogenic injury during surgical or radiological intervention. Accordingly, all the variants are very important in radiology and vascular surgery, as well as in planning operations in cardiothoracic, neck, and head surgery.

\section{ACKNOWLEDGMENTS}

This work was supported by grant No. 175061 from the Ministry of Science, Serbia. We are very grateful to Ms. Elza Holt for revising the English text of our manuscript.

\section{REFERENCES}

1. Atkin GK, Grieve PP, Vattipally VR, Ravikumar KH, Das SK (2007) The surgical management of aortic root vessel anomalies presenting in adults. Ann Vasc Surg, 21: 525-534.

2. Barbee JP, Stevens SL, Gaines TE, Gash JR (2007) Endovascular repair of cervical aortic arch aneurysm. Vasc Endovascular Surg, 41: 355-357.

3. Best IM, Bumpers HL (2002) Anomalous origins of the right vertebral, subclavian, and common carotid arteries in a patient with a four-vessel aortic arch. Ann Vasc Surg, 16: 231-234.

4. Bhatia K, Ghabriel MN, Henneberg M (2005) Anatomical variations in the branches of the human aortic arch: a recent study of a South Australian population. Folia Morphol, 64: 217-224. 
5. Cetin I, Varan B, Orün UA, Tokel K (2009) Common trunks of the subclavian and the vertebral arteries: presentation of a new aortic arch anomaly. Ann Vasc Surg, 23: 142-143.

6. Chahwan S, Miller MT, Kim KA, Mantell M, Kirksey L (2006) Aberrant right subclavian artery associated with a common origin of carotid arteries. Ann Vasc Surg, 20: 809-812.

7. Chan YC, Cheng SW, Ting AC, Ho P (2008) Supra-aortic hybrid endovascular procedures for complex thoracic aortic disease: single center early to midterm results. J Vasc Surg, 48: 571-579.

8. Cheng M, Xiadong X, Wang C, You C, Mao B, He M, Zhang $C$ (2009) Two anatomic variations of the vertebral artery in four patients. Ann Vasc Surg, 23: 689, e1-5.

9. Cinŕ CS, Arena GO, Bruin G, Clase CM (2000). Kommerell's diverticulum and aneurysmal right-sided aortic arch: A case report and review of literature. J Vasc Surg, 32: 1208-1214.

10. Epstein DA, Debord JR. 2002. Abnormalities associated with aberrant right subclavian arteries-a case report. Vasc Endovascular Surg, 36: 297-303.

11. Fazan VP, Caetano AG, Filho OA (2004) Anomalous origin and cervical course of the vertebral artery in the presence of a retroesophageal right subclavian artery. Clin Anat, 17: 354-357.

12. Fazan VPS, Ribeiro RA, Ribeiro JAS, Filho OAR (2003) Right retroesophageal subclavian artery. Acta Cirurg Bras, 18 (suppl. 5): 54-56.

13. Floemer F, Magerkurth $O$, Jauckus $C$, Lütschg J, Schneider JF (2008) Klippel-Feil syndrome and sprengeld deformity combined with an intraspinal course of the left subclavian artery and a bovine aortic arch variant. Am J Neuroradiol, 29: 306-307.

14. Fong LV, Venables AW (1987) Isolation of the left common carotid or left innominate artery. Br Heart J, 57: 552-554.

15. Gluncic V, Ivkic G, Marin D, Percac S (1999) Anomalous origin of both vertebral arteries, Clin Anat, 12: 281-284.

16. Goray VB, Joshi AR, Garg A, Merchant S, Yadav B, Maheshwari $P$ (2005) Aortic arch variation: a unique case with anomalous origin of both vertebral arteries as additional branches of the aortic arch distal to left subclavian artery. Case report. Am J Neuroradiol, 26: 93-95.

17. Higashi N, Shimada H, Simamura E, Hatta T (2008) Right vertebral artery as the fourth branch of the aortic arch. Anat Sci Int, 83: 314-318.

18. Ito H, Konishi A, Kon-Nai T, Ishibashi T, Takahashi S (2006) Double aortic arch with atresia, tapering and aneurysm of the left arch. Br J Radiol, 79: e71-e74.

19. Jayanthi V, Prakash L, Devi MN, Geethanjali BS, Rajini T (2010) Anomalous origin of the left vertebral artery from the arch of the aorta: review of the literature and a case report. Folia Morphol, 69: 258-260.

20. Komiyama M, Morikawa T, Nakajima H, Nishikawa M, Yasui T (2001) High incidence of arterial dissection associated with left vertebral artery of aortic origin. Neurol Med Chir, 41: 8-12.

21. Kumar S, Pamidi N, Somayaji SN, Nayak S, Vollala VR (2010) Anomalous branching pattern of the aortic arch and its clinical applications. Singapore Med J, 51: e182-e183.

22. Lemke A-J, Liebig T, Felix R (1999) Anomalous origin of the right vertebral artery: Review of the literature and case report of right vertebral artery origin distal to the left subclavian artery. Am J Neuroradiol, 20: 1318-1321.

23. McElhinney DB, Hoydu AK, Gaynor JW, Spray TL, Goldmuntz E, Weinberg PM (2001a) Patterns of right aortic arch and mirror-image branching of the brachiocephalic vessels without associated anomalies. Pediatr Cardiol, 22: 285-291.

24. McElhinney DB, McDonald-McGinn D, Zackai EH, Goldmuntz E (2001b) Cardiovascular anomalies in patients diagnosed with a chromosome 22q11 deletion beyond 6 months of age. Pediatrics, 108: E104.

25. Nathan H, Seidel MR (1983) The association of a retroesophageal right subclavian artery, a right-sided terminating thoracic duct, and a left vertebral artery of aortic origin: anatomical and clinical considerations. Acta Anat, 117: 362-373.

26. Natsis K, Didagelos M, Manoli SM, Papathanasiou E, Sofidis G, Anastasopoulos N (2011) A bicarotid truk in association with an aberrant right subclavian artery. Report of two cases, clinical impact, and review of the literature. Folia Morphol, 70: 68-73.

27. Natsis KI, Tsitouridis IA, Didagelos MV, Fillipidis AA, Vlasis KG, Tsikaras PD (2009) Anatomical variations in the branches of the human aortic arch in 633 angiographies: clinical significance and literature review. Surg Radiol Anat, 31: 319-323.

28. Nayak SR, Pai MM, Prabhu LV, D'Costa S, Shetty P (2006) Anatomical organization of aortic arch variations in the India: embryological basis and review. J Vasc Bras, 5: 95-100.

29. Nelson ML, Sparks CD (2001) Unusual aortic arch variation: distal origin of common carotid arteries. Clin Anat, 14: 62-65.

30. Park JK, Kim SH, Kim BS, Choi G (2008) Two cases of aberrant right subclavian artery and right vertebral artery that originated from the right common carotid artery. Korean J Radiol, 9 (suppl.): S39-S42.

31. Rusu MC, Boşcu AL (2010) Transverse subisthmic course of the innominate artery in an adult: detailed anatomy and additional variation. Folia Morphol, 89: 261-266.

32. Sadler TW (2006) Cardiovascular system. In: Sadler TW ed.. Langman's medical embryology. 10th Ed. Lippincott Williams Wilkinson, a Wolters Kluwer business, Philadelphia, pp. 159-194.

33. Schwarzacher SW, Krammer EB (1989) Complex anomalies of the human aortic arch system: unique case with both vertebral arteries as additional branches of the aortic arch. Anat Rec, 225: 246-250.

34. van Son JA, Julsrud PR, Hagler DJ, Sim EK, Pairolero PC, Puga FJ, Schaff HV, Danielson GK (1993) Surgical treatment of vascular rings: the Mayo Clinic experience. Mayo Clin Proc, 68: 1056-1063.

35. Weinberg PM (2006) Aortic arch anomalies. J Cardiovasc Magn Reson, 8: 633-643. 